Please quote as: Bretschneider, U.; Heider, A.; Rüsen, T. \& Hülsbeck, M. (2019): Diffusion von digitalen Technologien und Trends in Familienunternehmen. In: Kollmann, T. (Ed.), Handbuch Digitale Wirtschaft. Wiesbaden, Germany: Springer Gabler. 


\title{
Diffusion von digitalen Technologien und Trends in Familienunternehmen
}

\author{
Ulrich Bretschneider, Anne Heider, Tom Rüsen und \\ Marcel Hülsbeck
}

\section{Inhalt}

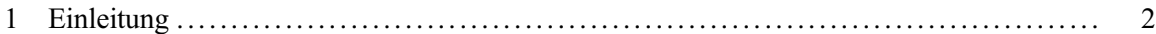

2 Theoretischer Bezugsrahmen ............................................ 3

3 Diffusion von digitalen Technologien und Trends in Familienunternehmen ............ 5

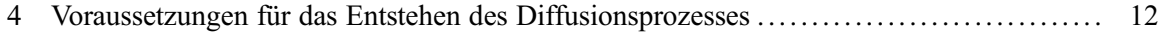

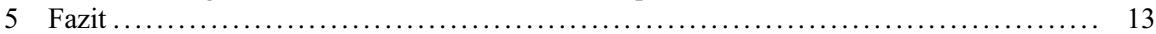

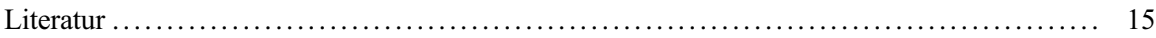

\section{Zusammenfassung}

Der vorliegende Beitrag beschreibt den Diffusionsprozess von digitalen Technologien und Trends in Familienunternehmen (FU). Die Diffusion von digitalen Technologien und Trends in FU gestaltet sich wegen des Einfluss der Eigentümerfamilie auf das FU im Vergleich zu Nicht-FU grundlegend anders. Eigenschaften der Familie sowie des FU und der digitalen Technologien und Trends wirken sich zugleich negativ auf die Geschwindigkeit, mit der digitale Technologien und Trends den Diffusionsprozess im FU durchlaufen, aus. Dagegen entwickeln FU spezifische Strategien, die dabei helfen, diese Zeitbarrieren zu umgehen.

\section{Schlüsselwörter}

Digitale Technologien · Digitale Trends · Digitalisierung · Diffusion · Familienunternehmen

U. Bretschneider $(\bowtie) \cdot$ A. Heider $\cdot$ T. Rüsen $\cdot$ M. Hülsbeck

Wittener Institut für Familienunternehmen (WIFU), Universität Witten/Herdecke, Witten, Deutschland

E-Mail: ulrich.bretschneider@uni-wh.de; anne.heider@uni-wh.de; tom.ruesen@uni-wh.de; marcel.huelsbeck@uni-wh.de 


\section{$1 \quad$ Einleitung}

Digitale Technologien und Trends bergen hohe Potenziale für Unternehmen. Mit ihrer Hilfe können Prozesse effizienter sowie Produkte und Dienstleistungen kundenfreundlicher gestaltet werden. Unter digitalen Technologien fasst man einerseits Hardware und Netzstrukturen und andererseits Anwendungen zusammen (Stief et al. 2016). Beispiele für digitale Technologien sind Sensoren, Virtual Reality-Systeme, Künstliche Intelligenz etc. Digitale Technologien können durch ihr inhärentes Potenzial digitale Trends hervorbringen (Bharadwaj et al. 2013). Unter einem digitalen Trend versteht man beispielsweise das Konzept „Industrie 4.0“ (Bharadwaj et al. 2013), was eine weitestgehend selbstorganisierte Produktion bezeichnet. Dies wird dadurch erreicht, dass zur Herstellung von Produkten eine Kombination aus intelligent vernetzten digitalen Technologien mit Facharbeitern kooperiert und dafür die digitalen Technologien untereinander aber auch mit den Facharbeitern kommunizieren. Dieses sozio-technische System, also die Kombination von digitalen Technologien mit sozialen Akteuren, macht das Wesen von digitalen Trends aus. Ein weiteres Beispiel für einen digitalen Trend ist das Crowdsourcing. Darunter versteht man, dass Unternehmen kleinere Aufgaben an eine undefinierte Masse an Menschen, die Crowd (Unternehmen und die Crowd als soziale Akteure), auslagert; und zwar über das Internet (Netzstruktur) mit der Hilfe einer speziellen InternetPlattform (Anwendung) (Blohm und Bretschneider 2016). In diesem Sinne können Konzepte wie „Big Data“ oder das „Cloud Computing“ ebenfalls als digitale Trends bezeichnet werden.

Digitale Technologien und Trends finden immer über zwei mögliche Wege den Eingang ins Unternehmen (Scheer 2016; Stief et al. 2016). Einerseits werden sie eingesetzt, um Prozesse oder Abläufe im Unternehmen zu unterstützen (Stief et al. 2016). Dies können sowohl Kernprozesse, wie zum Beispiel der zentrale Produktionsprozess in einem klassischen Industriebetrieb, als auch verschiedene Supportprozesse im Unternehmen sein. Ziel einer Digitalisierung von Prozessen ist es, diese Prozesse effizienter hinsichtlich der Parameter Zeit, Qualität und/oder Kosten zu gestalten (Scheer 2016). Einen umso größeren Effizienznutzen generieren Unternehmen dabei, wenn es für die Unternehmenswertschöpfung relevante Kernprozesse oder gar damit zusammenhängende, unternehmensweite Prozesslandschaften sind, die digitalisiert werden. Andererseits werden digitale Technologien und Trends in Unternehmen dazu genutzt, um bestehende Produkte und Dienstleistungen zu digitalisieren, also diese mit digitalen Technologien und Trends nachträglich auszustatten, oder um damit gänzlich neue Produkte und Dienstleistungen zu innovieren (Stief et al. 2016). Ziel einer solchen Produkt- und Dienstleistungsdigitalisierung ist es, einen höheren Kundennutzen und/oder ein höheres Produkt- und Dienstleistungserlebnis zu generieren (Scheer 2016).

Für die Digitalisierung von Produkten, Dienstleistungen und Prozessen werden in Unternehmen digitale Technologien und Trends systematisch identifiziert, geprüft, ausgewählt und implementiert (Demary et al. 2016). Dieser Prozess wird im Allgemeinen als Diffusion bezeichnet, welche die Ausbreitung von materiellen und immateriellen Objekten in sozialen Systemen erklärt (Corsten et al. 2016). Dabei 
stellt die Zeit einen entscheidenden Faktor in solchen Diffusionsprozessen von digitalen Technologien und Trends dar (Demary et al. 2016). Je schneller es Unternehmen gelingt, digitale Technologien und Trends in ihren Produkten, Dienstleistungen und Prozessen zu implementieren und daraus wie oben beschrieben einen Nutzen in Form von Effizienzvorteilen oder Erhöhung des Kundennutzens zu generieren, desto schneller können sie sich auf diese Weise einen Wettbewerbsvorteil gegenüber der Konkurrenz aufbauen oder einen bestehenden Wettbewerbsvorteil von Konkurrenten einholen. Vor diesem Hintergrund sind Unternehmen bemüht, geeignete digitale Technologien und Trends möglichst schnell zu diffundieren.

Dies gilt auch für Familienunternehmen. Wegen der besonderen Rolle der Familie und ihren Einfluss auf das Familienunternehmen - zum Beispiel in ihrer Rolle als Eigentümer des Familienunternehmens - gestaltet sich der Diffusionsprozess von digitalen Technologien und Trends allerdings im Vergleich zu NichtFamilienunternehmen grundlegend anders. Eigenschaften der Familie sowie des Familienunternehmens und der digitalen Technologien und Trends wirken sich zugleich in Bezug auf den Faktor Zeit hemmend auf den Diffusionsprozess aus. Allerdings entwickeln Familienunternehmen spezifische Strategien, die dabei helfen, diese Zeitbarrieren zu umgehen oder zumindest zu verringern.

Der vorliegende Beitrag beschreibt den Diffusionsprozess von digitalen Technologien und Trends in Familienunternehmen. Im Beitrag wird zudem aufgezeigt, welche Eigenschaften der Familie, des Familienunternehmens und der digitalen Technologien und Trends sich negativ bzw. hemmend auf einen zügigen Durchlauf des Diffusionsprozess auswirken und welche Strategien Familienunternehmen gegen diese Negativfaktoren entwickeln, um eine Diffusion in möglichst kurzer Zeit zu durchlaufen.

\section{Theoretischer Bezugsrahmen}

\subsection{Familienunternehmen}

Familienunternehmen haben nicht nur in Deutschland, sondern ebenso weltweit eine große volkswirtschaftliche Bedeutung (Anderson und Reeb 2004; Astrachan und Shanker 2003; Haunschild und Wolter 2010). In Deutschland gehören gemäß einer Studie des Institutes für Mittelstandsforschung Bonn ca. $95 \%$ der Unternehmen zur Kategorie der Familienunternehmen, welche rund $61 \%$ aller sozialversicherungspflichtigen Beschäftigten anstellen (Haunschild und Wolter 2010).

Zur Beantwortung der Frage, was ein Familienunternehmen ausmacht bzw. was ein Familienunternehmen von einem Nicht-Familienunternehmen unterscheidet, werden in der Familienunternehmensforschung zahlreiche Charakteristiken und Definitionsansätze herangezogen. Diese beziehen sich u. a. auf den Grad des Eigentums und/oder der Führung des Familienunternehmens durch die Eigentümerfamilie, den Grad der Einbindung der Eigentümerfamilie in das Familienunternehmen oder es handelt sich um eine Kombination der angeführten Charakteristika (Handler 1989). Ein weit verbreiteter Ansatz zur Definition von Familienunternehmen ist 
der Components of involvement approach (Chrisman et al. 2005, 2012; Chua et al. 1999). Danach ist die Einbindung einer Familie in ein Unternehmen hinreichend, um ein Unternehmen als Familienunternehmen zu klassifizieren (Chrisman et al. 2005; Chua et al. 1999). Einbindung manifestiert sich dabei vor allem durch den Grad der Einbindung der Familie in das Management und die Governance des Unternehmens, wobei sich das Ausmaß der Einbindung in der Regel danach richtet, in welchem Eigentumsverhältnis die Familie zum Unternehmen steht. Charakteristisch für ein Familienunternehmen ist, dass diese Einbindung der Familie die Formulierung, Gestaltung und Implementierung der Firmenziele, -strategien, -strukturen beeinflusst und sogar prägen (Chua et al. 1999), denn in der Beeinflussung spiegeln sich die Ziele, Wünsche und Präferenzen der Familie wider (Sieger und Zellweger 2012; von Schlippe et al. 2017; Wimmer et al. 2011). Deshalb wird die Unternehmerfamilie in dieser Hinsicht sogar als ,,identitätsstiftendes Merkmal“ von Familienunternehmen bezeichnet wird (Wimmer et al. 2011, S. 19).

In dieser Hinsicht können die Einbindung und die damit verbundene Einflussnahme der Familie über verschiedene Kanäle und in verschiedenen Formen erfolgen. So kann die Einbindung und Einflussnahme der Familie einerseits direkt in der Form geschehen, dass eine bestimmte Anzahl von Familienmitgliedern die Geschäftsleitung übernimmt (Berrone et al. 2012). Denkbar wäre in dieser Hinsicht beispielsweise eine Gruppe von Geschwistern, die das Familienunternehmen in der zweiten Generation als Geschäftsführer gemeinschaftlich leitet. Eher indirekt folgt die Einbindung und Einflussnahme über die Mitgliedschaft der Familienmitglieder in kontrollierenden und beratenden Gremien des Familienunternehmens, bspw. dem Aufsichts- oder Beirat. Die operative Geschäftsführung hat in diesem Fall dann ein familienexternes Managementteam oder ein externer Management inne. Dieses Management wird dann aber von der Familie in ihrer Aufsichts- oder Beiratsrolle in seinen Entscheidungen beeinflusst (Berrone et al. 2012).

In beiden Rollen ist der Grad des Einflusses der Familienmitglieder dann nicht nur über ihre Eigentumsverhältnisse, sondern darüber hinaus auch durch ihre Persönlichkeitsmerkmale und Eigenschaften charakterisiert, beispielsweise durch eine starke Stellung als Eigentümer des Familienunternehmens, durch ein ausgeprägtes Charisma eines involvierten Familienmitglieds oder durch einen zugeschriebenen Status im Familienunternehmen (Berrone et al. 2012).

\subsection{Diffusionstheorie nach Rogers}

Diffusionstheorien beschreiben im Allgemeinen, wie sich Innovationen in einem sozialen System verbreiten und angenommen werden. Als Innovation gelten dabei alle Ideen, Prozesse und Objekte, die für eine soziale Gruppe subjektiv als neu wahrgenommen werden (Corsten et al. 2016). Auf Mikroebene stellen dabei Individuen und Gruppen in einer Größe von zwei bis ca. 10 Mitgliedern die Untersuchungsobjekte dar (Corsten et al. 2016). Eine der bedeutendsten Theorien, die die Diffusion durch Individuen und Gruppen prozesshaft beschreibt, ist die von Rogers (2003). Rogers (2003) beschreibt die Übernahme von Innovationen als einen sozia- 


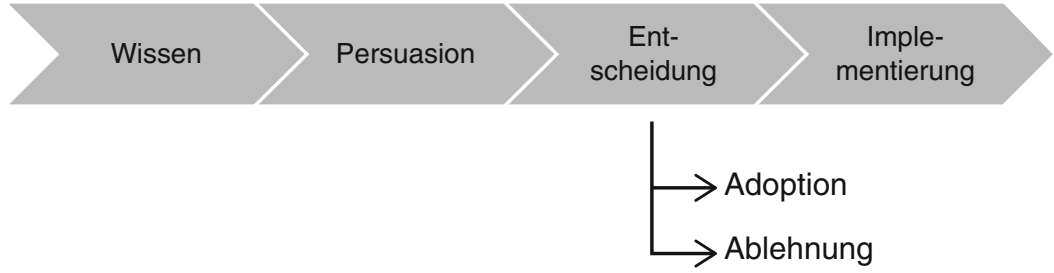

Abb. 1 Diffusionsprozess nach Rogers (2003)

len Prozess, der sich über einen bestimmten Zeitraum erstreckt und eine Reihe von auf einander aufbauenden Handlungen, also als ein kumulativer Prozess zu verstehen ist (Corsten et al. 2016), impliziert. Diesen fünfstufigen Prozess zeigt Abb. 1.

In der ersten, als Wissen (knowledge) bezeichneten Phase, erfährt das Individuum von der Existenz der Innovation und entwickelt ein Verständnis dafür, wie diese funktioniert. Rogers (2003, S. 172-173) differenziert dieses Wissen weiter aus und unterscheidet zwischen awareness-knowledge, how-to-knowledge und principlesknowledge. Während ersteres das Wissen um die schiere Existenz einer Innovation beschreibt, ermöglicht die zweite Form die korrekte Anwendung dieser. Das principles-knowledge schließlich beschreibt das Grundlagenwissen (prinzipielles Wissen zur Funktionsweise) über eine Innovation und ist für die Adoption an sich nicht notwendig. Jedoch steigt mit fehlendem Grundlagenwissen die Wahrscheinlichkeit, die Innovation falsch zu verwenden und die Nutzung folglich $\mathrm{zu}$ beenden (Rogers 2003, S. 173).

Die zweite Phase wird als Persuasion (persuasion) bezeichnet und beschreibt das Stadium, in dem das Individuum eine bestimmte Einstellung gegenüber der Innovation entwickelt und die möglichen Folgen einer (Nicht-)Übernahme abwägt (Rogers 2003, S. 177-179). Die Person oder Gruppe entwickelt Vorstellungen darüber, wie die Innovation in ihrem subjektiven Kontext eingesetzt werden könnte. Im Vordergrund steht die Frage, welche Vor- und Nachteile sich daraus ergeben.

In der dritten Phase (decision) wird die tatsächliche Entscheidung zur Übernahme oder Ablehnung der Innovation getroffen und somit die zuvor gebildete Einstellung in konkretes Handeln überführt (Rogers 2003, S. 178). Im positiven Falle folgt nach der Entscheidung die Implementierung (implementation) als vierte Phase im Innovations-Entscheidungs-Prozess (Rogers 2003, S. 179-188). Erst an dieser Stelle erfolgt die tatsächliche Verwendung der Innovation durch das Individuum.

\section{Diffusion von digitalen Technologien und Trends in Familienunternehmen}

Wie dargelegt, entwickeln Familien in ihrer so genannten Family Governance (Koeberle-Schmid und Nützel 2005, S. 22; Witt 2008) Ziele, Wünsche und Präferenzen hinsichtlich der Ausrichtung des Familienunternehmens. Auf Grund ihrer Eigentumsverhältnisse und/oder ihrer Persönlichkeitsmerkmale, durch ein ausgeprägtes Charisma 
oder einem ihr zugeschriebenen Status übt die Familie dann Einfluss auf die Formulierung, Gestaltung und Durchsetzung der Firmenziele, -strategien und -strukturen, die Führung und Führungsstruktur sowie die Entscheidungsprozesse und -strukturen, also die Corporate Governance des Familienunternehmens (Koeberle-Schmid und Nützel 2005, S. 22; Witt 2008), aus.

Diese Form der Einflussnahme durch die Familie geschieht ebenso in Bezug auf die Übernahmeentscheidungen von digitalen Technologien und Trends im Familienunternehmen. Konkret nimmt die Familie hierbei einzelne digitale Technologien oder digitale Trends in Augenschein, welche dann einen familieninternen Diffusionsprozess durchlaufen. Dabei steht die Frage im Vordergrund, inwieweit die betrachtete Technologie oder der betrachtete Trend einen Nutzen im Familienunternehmen stiften kann oder nicht. Im Rahmen des familieninternen Diffusionsprozesses wird also zunächst im Kreise der Familienmitglieder darüber diskutiert, ob eine betreffende Technologie bzw. ein betreffender Trend sich im Kontext des eigenen Familienunternehmens dazu eignet, einen bestimmten Prozess zu optimieren oder den Nutzen eines bestehenden Produktes zu erweitern. Im weiteren Verlauf dieses Diffusionsprozesses kommt es dann im Familienrat zu einer Übernahmeentscheidung oder einer Ablehnung der betreffenden Technologie bzw. des betreffenden Trends. Im Falle einer Übernahmeentscheidung wird es dann letztlich zu einer Implementation der betreffenden Technologie bzw. des betreffenden Trends in den Strukturen bzw. Produkten des Familienunternehmens kommen. Das bedeutet, dass die Wissens-, Persuasions- und Entscheidungsphase der Family Governance unterliegen, wogegen es dann im Übergang zur Implementierungsphase zu einer aktiven Einflussnahme der Familie auf das Familienunternehmen kommt. Die Implementierung der im Fokus stehenden digitalen Technologien oder Trends (Implementierungsphase) erfolgt dann im Rahmen der Corporate Governance. Abb. 2 verdeutlicht diesen Zusammenhang.

Dieser grob skizzierte Diffusionsprozess wird nachfolgend in seinen einzelnen Phasen ausführlich beschrieben.

\subsection{Wissensphase}

In dieser Phase des Diffusionsprozesses verfolgt die Familie das Ziel, Wissen über digitale Technologien und Trends zu erlangen. Dabei geht es einerseits darum, im Kreise der Familie zu klären, welche digitalen Technologien und Trends es überhaupt gibt. Es wird also ein Bewusstsein über die Existenz bestimmter digitaler Technologien und Trends aufgebaut, was gemäß Rogers (2003) dem Aufbau von awareness-knowledge entspricht. Andererseits geht es in dieser Phase auch darum, im Familienkreis grundlegendes Verständnis sowie tieferliegendes Detailwissen darüber aufzubauen, wie eine dann konkret in Augenschein genommene digitale Technologie (bzw. digitaler Trend) funktioniert und angewendet wird (How-toknowldege und Principles-knowledge gemäß Rogers 2003).

Der Aufbau von How-to- und Principles-knowledge hängt dabei maßgeblich vom Vorwissen der einzelnen Familienmitglieder ab. Vorwissen meint, inwieweit die 


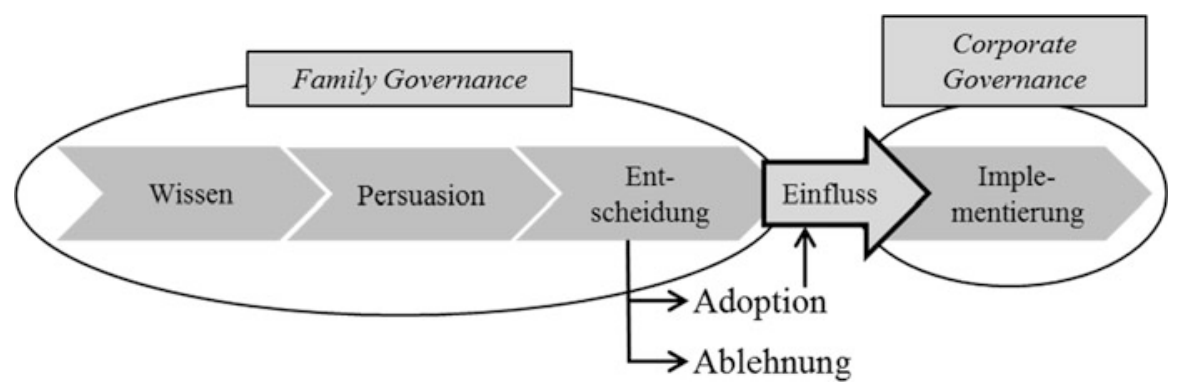

Abb. 2 Diffusionsprozess von digitalen Technologien und Trends in Familienunternehmen. (Quelle: Eigene Darstellung in Anlehnung an Rogers (2003))

einzelnen Familienmitglieder ein Grundverständnis und Erfahrungen aus dem Bereich des How-to-knowldege und Principles-knowledge für die im Fokus stehende digitale Technologie (bzw. digitaler Trend) verfügt. Ist das Vorwissen bei mindestens einem Familienmitglied bereits ausgeprägt, so wird dieses Mitglied sein Wissen mit den anderen Familienmitgliedern teilen, um alle Familienmitglieder auf denselben Wissensstand zu bringen. Die Anzahl der Familienmitglieder beeinflusst dabei, wie lange es dauert, um alle Familienmitglieder auf denselben Wissensstand zu bringen. So wird dies umso mehr Zeit in Anspruch nehmen, je größer der Kreis der Familienmitglieder ist.

Verfügt dagegen keines der Familienmitglieder über ausreichendes How-to- und Principles-knowledge in Bezug auf die im Fokus stehende digitale Technologie (bzw. digitaler Trend), so werden sich die einzelnen Familienmitglieder dieses Wissen jeweils selber aneignen. Häufig sind es ältere Familienmitglieder, die nicht der so genannten Generation der „Digital Natives“, also der gesellschaftlichen Generation, die in der digitalen Welt aufgewachsen ist, entstammen (Palfrey und Gasser 2008), die wenig Vorwissen mitbringen (Bretschneider et al. 2019).

Der Wissensaufbau der einzelnen Familienmitglieder hängt dabei von der Komplexität der digitalen Technologie (bzw. digitaler Trend) ab. Beispielsweise stellt die digitale Technologie der Künstlichen Intelligenz ein hochkomplexes Thema dar, dessen Durchdringung und damit der Aufbau von How-to- und Principles-knowledge hierzu relativ viel Zeit in Anspruch nimmt.

Ist kein Vorwissen unter den Familienmitgliedern vorhanden und handelt es sich bei der im Fokus stehenden digitalen Technologie (bzw. digitaler Trend) um ein komplexes Phänomen, kommt es häufig vor, dass zur Unterstützung des Wissensaufbaus Familien-externe Experten mit entsprechender Digitalisierungsexpertise zur Rate gezogen werden. In diesen Fällen würde ein eigener, d. h. ohne fremde Hilfe, arrangierter Wissensaufbau überproportional viel Zeit in Anspruch nehmen, weshalb ein schnellerer Zugang zu diesem Wissen eben über diese familien-externen Wissensquellen gesucht wird. Insbesondere nachfolgend beschriebene Wissensquellen werden genutzt:

Stabstelle „Digitalisierung “: Im Allgemeinen versteht man in der Managementliteratur unter einer Stabsstelle eine spezialisierte Leitungshilfestelle, der ein definiertes, 
fachliches Aufgabenspektrum zugeschrieben wird, dabei aber gleichzeitig keine Entscheidungs- und Weisungskompetenzen zugesprochen wird (Schmidt 2000). Stabsstellen werden üblicherweise von hoch qualifizierten Fachkompetenzen besetzt und sollen vor allem der Managementebene beratend zur Seite stehen, der sie zugeordnet sind. Durch die Beratungsleistungen des Inhabers dieser Stabstelle baut sich die Familie systematisch Wissen über digitale Technologien und Trends auf. Es wird einerseits ein Bewusstsein über die Existenz von digitalen Technologien und Trends geschaffen (awareness-knowledge). Andererseits hilft der Stabsstelleninhaber dabei, grundlegendes Verständnis sowie tieferliegendes Detailwissen über digitale Technologien und Trends aufzubauen (How-to-knowledge und Principles-knowledge).

Die Maßnahme der Einrichtung einer solchen Stabsstelle zur Unterstützung eines systematischen Wissensaufbaus der Familienmitglieder hat dabei einen nachhaltigen Charakter. So stellt dies sicher keine Maßnahme dar, um den Wissensaufbau in Bezug auf eine konkret ins Auge gefasste digitale Technologie (bzw. digitaler Trend) zu unterstützen. Vielmehr berät der Stabstelleninhaber auch in Bezug auf andere digitale Technologien und Trends, die den Diffusionsprozess der Familie durchlaufen werden. Auf Grund seines nachhaltigen Charakters wird diese Maßnahme des Wissensaufbaus eher von Familien verfolgt, deren Mitglieder per se ein geringes Grundverständnis in Bezug auf digitale Technologien und Trends sowie eine geringe Affinität hierfür aufweisen.

Digital Advisory Boards (Außenperspektive): Um im oben beschriebenen Sinne Wissen über digitale Technologien und Trends aufzubauen, richten Familien - egal ob sie das Familienunternehmen operativ als Geschäftsleitung führen oder in Kontroll- oder Aufsichtsgremien tätig sind - häufig auch so genannte Digital Advisory Boards (Digitalbeirat) ein. Im Allgemeinen ist ein solches Digital Advisory Board genauso wie Aufsichts- oder Beiratsgremien als kontrollierende und beratende Instanz in einem Unternehmen tätig, fokussiert seine Tätigkeiten aber (parallel zu denen des Aufsichts- oder Beirates) alleine auf die Digitalisierung im Unternehmen. Große Unternehmen, wie etwa der Reisekonzern Thomas Cook oder die Messe Frankfurt, fangen mehr und mehr an, ein solches Beratungsgremium zu installieren.

Ein Digital Advisory Board im Familienunternehmen setzt sich dabei sowohl aus erfahrenen Experten aus der Digitalwirtschaft (externe Mitglieder) als auch aus digitalisierungskompetenten Mitarbeitern aus dem eigenen Unternehmen (interne Mitglieder) zusammen, womit es sowohl eine Außen- als auch eine Innenperspektive einnimmt. In Bezug auf die Außenperspektive hat das Digital Advisory Board, und hier vor allem die externen Mitglieder, die Aufgabe, beratend digitale Trends zu antizipieren und $\mathrm{zu}$ reflektieren und daraus allgemeine Chancen und Gefahren abzuleiten. Durch die Tätigkeiten des Digital Advisory Boards baut sich die Familie also systematisch Wissen über aktuell existierende als auch zukünftig relevante digitale Technologien und Trends auf; sowohl Awareness-knowledge als auch How-to-knowldege und Principles-knowledge.

In Bezug auf die Innenperspektive obliegt es vor allem den internen Mitgliedern des Digital Advisory Boards, darüber zu reflektieren, wie digitale Technologien und Trends in den Kontext des Familienunternehmens eingesetzt werden könnten und welche Vor- und Nachteile sich daraus konkret für das Familienunternehmen ergeben. 
Diese Innenperspektive ist aber eher Gegenstand der Persuasionsphase, weshalb an dieser Stelle in Bezug auf die Innenperspektive auf das nachfolgende Unterkapitel verwiesen sei.

Die Einrichtung eines Digital Advisory Boards hat dabei einen ebenso nachhaltigen Charakter wie die oben beschriebene Maßnahme „Stabstelle“.

\subsection{Persuasionsphase}

In dieser Phase entwickelt und festigt die Familie eine grundsätzliche Einstellung oder Haltung gegenüber der im Fokus stehenden digitalen Technologien (bzw. digitaler Trend) auf der Basis des zuvor aufgebauten Wissens. Dabei geht es vor allem auch darum, im Familienkreis konkrete Vorstellungen darüber zu entwickeln, ob und wie die digitale Technologie (bzw. digitaler Trend) in den Kontext des eigenen Familienunternehmens eingesetzt werden könnte. In dieser Hinsicht wird man sich im Familienkreis zunächst die Frage stellen, ob die in Augenschein genommene digitale Technologie (bzw. digitaler Trend) beispielsweise in einen bestimmten Prozess implementierbar ist oder in ein Produkt einbaubar ist. Es geht also um die Kompatibilität der digitalen Technologie (bzw. digitaler Trend). Ist die Frage nach der Kompatibilität positiv beantwortet worden, so stellt sich die Frage, ob der Einsatz dieser digitalen Technologie auch einen konkreten Vorteil bzw. Nutzen bringt. So ist kritisch zu hinterfragen, ob der Einsatz einer digitalen Technologie in einem bestimmten Prozess auch einen Effizienzvorteil für diesen Prozess oder die Ausstattung eines Produktes mit einer Technologie für den Kunden einen Mehrwert mit sich bringt.

In kleineren Familienunternehmen, in denen die Familie operativ in die Geschäftsführung eingebunden ist, stellt die kritische Prüfung der Kompatibilität und Vorteile bzw. Nutzen für die Familie in der Regel keine Schwierigkeit dar, da die Familie in diesen Fälle im operativen Tagesgeschäft involviert ist und somit ganz konkrete Kenntnisse und Erfahrungen in Bezug auf die täglichen Routinen und Abläufe im eigenen Unternehmen hat. Je weiter die Familie allerdings vom operativen Tagesgeschäft entfernt ist und somit weniger über solche Kenntnisse und Erfahrungen verfügt - was beispielsweise dann der Fall ist, wenn das Familienunternehmen eine gewisse Größe erreicht hat oder die Familie sich auf die Besetzung von Aufsichts- und Beiratspositionen beschränkt - desto eher wird es im Familienkreis vorkommen, dass für die Einschätzung der Kompatibilität und Vorteile bzw. Nutzen Hilfe herangezogen werden muss.

In solchen Fällen sucht sich die Familie Hilfe über die Einrichtung eines Digital Advisory Boards. Oben wurde bereits die Einrichtung eines Digital Advisory Boards als eine Strategie der Wissensphase erläutert. Wie dargelegt, hat die Einrichtung eines Digital Advisory Boards in der Wissensphase zum Ziel, der Familie dabei zu helfen, sich Wissen über digitale Trends und Technologien anzueignen. In der Persuasionsphase geht es darum, mit der Hilfe dieses Boards Vorstellungen darüber zu entwickeln, wie digitale Technologien und Trends in das Familienunternehmen transferierbar sind. Hier kommen die internen Mitglieder des Advisory Boards zum 
Einsatz, da diese den Kontext des eigenen Unternehmens in der Regel sehr genau kennen und deshalb zuverlässig dabei helfen können, die Kompatibilität und Vorteile bzw. Nutzen einschätzen zu können. Insgesamt gesehen stellt die Arbeit der internen Mitglieder (Innenperspektive des Digital Advisory Boards) also die Grundlage dafür dar, dass sich die Familie eine grundsätzliche Einstellung oder Haltung gegenüber bestimmter digitaler Technologien und Trends aufbauen kann.

\subsection{Entscheidungsphase}

In dieser Phase wird im Familienkreis darüber entschieden, eine zur Diskussion stehende digitale Technologie (bzw. digitaler Trend) anzunehmen oder abzulehnen. Im Allgemeinen ist charakteristisch für diese Phase, dass obwohl der Adopter in den vorangegangenen Phase Wissen und Einstellung aufgebaut bzw. entwickelte hat, bezüglich der Innovation und damit der Annahme- oder Ablehnungsentscheidung immer noch Unsicherheiten existieren (Rogers 2003). Übertragen auf den Untersuchungskontext gilt dies auch für die Familie in Bezug auf eine zu Grunde liegende digitale Technologie (bzw. digitaler Trend). So entstehen Unsicherheiten in der Familie immer dann, wenn die im Fokus stehende digitale Technologie (bzw. digitaler Trend) zum einen hohe Anschaffungskosten und/oder zum anderen hohe Implementierungskosten verursachen würde. Wenn diese zudem in einem negativen Verhältnis zu dem in der vorangegangenen Phase abgeschätzten Nutzen bzw. Vorteilen stehen würden, steigt die Unsicherheit zusätzlich. Dementsprechend versuchen Familien in dieser Phase, Wege zu finden, mögliche Unsicherheit zu reduzieren, um somit die Entscheidung auf eine sicherere Basis zu stellen.

Um die Unsicherheiten zu reduzieren und somit mehr Sicherheit für die Annahme- oder Ablehnungsentscheidung zu schaffen, veranlassen Familien beispielsweise, Testumgebungen für zu Grunde liegende digitale Technologien und Trends innerhalb des Unternehmens einzurichten. Geht es beispielsweise darum, bestimmte Prozesse mittels digitaler Sensortechnik zu automatisieren oder bestehende Produkte mit bestimmten digitalen Technologien auszustatten, so werden solche Vorhaben typischerweise in Labor- oder Realumgebungen ausführlich getestet. Die Erkenntnisse aus dem Test dienen der Familie, darüber zu befinden, ob die getestete digitale Technologie bzw. der getestete digitale Trend dauerhaft im Familienunternehmen zum Einsatz kommt oder nicht.

Um die Unsicherheiten zu reduzieren, wählen Familien oftmals auch die Strategie, die Mitarbeiter in die Planungen bezüglich des Einsatzes von bestimmten digitalen Technologien und Trends zu involvieren, insbesondere dann, wenn diese der Prozessdigitalisierungen im Familienunternehmen dienen sollen. In solchen Fällen werden die Mitarbeiter ganz konkret dazu aufgefordert, in ihrem Arbeitsalltag aktiv zu prüfen und zu hinterfragen, inwieweit ins Auge gefasste digitale Technologien und Trends die eigenen Prozesse vereinfachen oder beschleunigen können. Darüber hinaus werden Mitarbeiter gebeten, Ideen und Gestaltungsvorschläge hinsichtlich einer Prozessoptimierung mit diesen digitalen Technologien und Trends zu unterbreiten. Das auf diese Weise von den Mitarbeitern rückgespielte Feedback und die konkreten 
Gestaltungsvorschläge reduzieren die Unsicherheiten auf der Seite der Familie, was umso relevanter ist, wenn es Prozesse betrifft, in denen die Familie im Unternehmensalltag weniger involviert ist.

\subsection{Implementierungsphase}

In dieser Phase wird die betrachtete digitale Technologie (bzw. digitaler Trend) nach einer in der vorangegangenen Phase getroffenen positiven Adoptionsentscheidung von der Familie in das Familienunternehmen integrieren und zur Anwendung gebracht. Das bedeutet, dass in dieser Phase des Diffusionsprozesses eine aktive Einflussnahme von Seiten der Familie auf das Familienunternehmen stattfindet, wovon dann die Unternehmensstrukturen und -mitarbeiter betroffen sind.

Beispielsweise weckt eine Digitalisierung von Prozessen, insbesondere den Kernprozessen unter solchen Mitarbeitern, die Beteiligte dieser Prozesse sind, gewisse Ressentiments. So fürchtet man das Neue, weil man nicht weiß, wie die neuen Technologien zu bedienen sind, weil der Einsatz neuer digitaler Trends, wie zum Beispiel das Crowdsourcing, mit Änderungen in der bewährten Arbeitsorganisation und den gewohnten Arbeitsabläufen verbunden ist oder die neuen Technologien und Trends die Belegschaft im schlimmsten Fall überflüssig machen könnte. Diese Furcht der Mitarbeiter ist in Familienunternehmen meist sehr viel ausgeprägter als in Nicht-Familienunternehmen. Dies bestätigen auch die Ergebnisse einer Studie von Spitzley und Prügl (2017), wonach die Akzeptanz der Mitarbeiter gegenüber der Digitalisierung als zweithöchste Herausforderung im Zuge der digitalen Transformation im Familienunternehmen begriffen wird. Ursächlich für das Verstärkte auftreten dieses Phänomens in Familienunternehmen ist die in der Literatur immer wieder hervorgehobene besondere Beziehung der Unternehmerfamilie zur Belegschaft. Diese Beziehung wird oftmals von Werten wie „Zusammengehörigkeit“" und „Familienbewusstsein“ geprägt; in der Kultur von Familienunternehmen gehören die Beschäftigten gar zur erweiterten Familie (Campopiano und De Massis 2015; Wimmer 2011). Umgekehrt genießt die Unternehmerfamilie auf Grund dieser Situation ein überdurchschnittlich hohes Vertrauen der Mitarbeiter (Eßer 2018; Pittino et al. 2016). Dieses ist sogar umso höher, je stärker die Familie in das Unternehmen eingebunden ist (Berrone et al. 2012).

Vor diesem Hintergrund erscheint es umso plausibler, dass Familien die Implementierung digitaler Technologien und Trends im Rahmen eines systematischen Changemanagements, bei dem die Mitarbeiter in den Mittelpunkt gestellt werden, betreiben. Dabei werden die betroffenen Mitarbeiter nicht nur frühzeitig auf die anstehenden Veränderungen durch umfassende und angemessene Information vorbereitet, sondern auch zunehmend in die Gestaltung der Veränderung einbezogen („Ikea-Prinzip“) (Hehn et al. 2016). Fordert man die Mitarbeiter auf, in ihren Arbeitsalltag aktiv zu prüfen, inwieweit digitale Technologien und Trends die eigenen Prozesse vereinfachen oder beschleunigen können, und fordert man die Mitarbeiter darüber hinaus auf, Gestaltungsvorschläge für eine Prozessoptimierung mit der Hilfe von digitalen Technologien und Trends zu unterbreiten, dann stellt dies 
nicht nur eine Wertschätzung gegenüber den Mitarbeitern dar, sondern beugt darüber hinaus auch der Entwicklung von Ängsten und Ressentiments sowie dem Abbau von Vertrauen vor (Bretschneider et al. 2019). Damit vermittelt man den betroffenen Mitarbeitern die nötige Sicherheit (Hehn et al. 2016). Je stärker diese Sicherheit, desto größer die Bereitschaft zur Veränderung (Hehn et al. 2016). Wenn diese Bereitschaft nicht erzeugt wird, können Widerstände aus der Belegschaft die Digitalisierung zum Scheitern bringen (Hehn et al. 2016).

\section{$4 \quad$ Voraussetzungen für das Entstehen des Diffusionsprozesses}

Im vorangegangenen Kapitel wurden nicht nur die einzelnen Phasen des Diffusionsprozesses erläutert, sondern auch für jede einzelne Phasen mögliche Faktoren, die den Durchlauf des Prozesses negativ beeinflussen können, vorgestellt. Darüber hinaus gibt es in Bezug auf die Eigenschaften der Familie gewisse Voraussetzungen, die erfüllt sein müssen, um eine Diffusion von digitalen Technologien und Trends im Familienunternehmen überhaupt erst entstehen zu lassen. Auf diese soll in diesem Kapitel näher eingegangen werden.

Der Grad der Offenheit gegenüber der Digitalisierung im eigenen Unternehmen stellt in diesem Sinne eine Voraussetzung für die Digitalisierung dar (Bretschneider et al. 2019). Beispielsweise können subjektiv-emotionale Einstellungen, wie etwa mangelnde Risikobereitschaft, fehlende Relevanzeinschätzung, Angst vor Strukturveränderungen, Trägheit oder Abneigung gegenüber Neuerungen, bei einzelnen Familienmitgliedern zu einer geringen Offenheit gegenüber der Digitalisierung des eigenen Unternehmens führen. Oftmals weisen insbesondere Senioren in der Familie eine geringe Offenheit auf. Diese Mitglieder der Unternehmerfamilie sind in ihren Perspektiven auf Markt- und Wettbewerbsdynamiken sowie adäquaten Organisationskonzepten und Geschäftsmodelldesigns erheblich durch Zusammenhänge geprägt worden, in denen die Chancen und Risiken der Digitalisierung nicht existent waren (Bretschneider et al. 2019; Rüsen et al. 2019). Gegenüber der so genannten Generation der „Digital Natives“, also der gesellschaftlichen Generation, die in der digitalen Welt aufgewachsen ist, fehlt ihnen oftmals die Fähigkeit, die sich potenzierenden Dynamisierungsgrade zu erfassen und entsprechende Maßnahmen zur Chancenverwertung bzw. Risikoabwendung zu ergreifen. Oftmals auch in Kombination mit großen unternehmerischen Erfolgen in der prä-digitalen Wirtschaftsära ist diese Generation tendenziell weniger offen gegenüber dem Thema der Digitalisierung, und beäugt es sogar eher skeptisch (Palfrey und Gasser 2008). Dieser beobachtbare Zusammenhang deckt sich mit empirischen Erkenntnissen einer Studie der Unternehmensberatung Pricewaterhouse Coopers aus dem Jahr 2017. Im Rahmen dieser Studie wurde die Generation der Senioren in Unternehmerfamilien befragt. 83 Prozent gaben an, dass Sie das Thema Digitalisierung aus den oben genannten Gründen weniger relevant empfinden (Wills 2017).

Weisen vereinzelte Familienmitglieder einen geringen Grad der Offenheit gegenüber dem Thema der Digitalisierung auf, so kann dies das Zustandekommen einer 
Diffusion überhaupt erst verhindern, wenn es auf der anderen Seite Familienmitglieder gibt, die einen hohen Grad der Offenheit mitbringen. Häufig sind dies jüngere Familienmitglieder aus der Generation der „Digital Natives“, also der gesellschaftlichen Generation, die in der digitalen Welt aufgewachsen ist. Denn so kommt es in solchen Situationen oftmals zwischen Senioren und der Nachfolgegeneration zu großen Meinungsverschiedenheiten. Die Existenz dieses Konfliktpotenzials wird durch die Studie von Wills (2017) belegt: So sagen 40 Prozent der befragten Mitglieder aus der NextGen aus 31 Ländern, dass sie frustriert seien, weil sie mit ihren Ideen zur Digitalisierung bei den Senioren nicht durchdringen (Wills 2017). Ähnlich lauten die Zahlen aus einer Studie von Spitzley und Prügl (2017), wonach lediglich 28 Prozent der Vertreter der jüngeren Generation mit dem Stand der Digitalisierung im eigenen Haus zufrieden sind. Der Rest der Befragten zeigt sich eher unzufrieden und wünscht sich von der Seniorgeneration in der Familie mehr Offenheit in Bezug auf die Digitalisierung im eigenen Unternehmen (Spitzley und Prügl 2017). Eine weitere Studie von Schlaadt (2017) zeichnet ein ähnliches Bild: Hier liegen in 64 Prozent der untersuchten Fälle Generationskonflikte in Bezug auf die Digitalisierung im eigenen Familienunternehmen vor. Die mangelnde Offenheit der Senioren innerhalb der Unternehmerfamilien gegenüber der Digitalisierung im eigenen Hause wurde dabei als maßgebliche Konfliktursache benannt (Schlaadt 2017). Insgesamt gesehen haben unterschiedliche Ausprägungen der Offenheit einzelner Familienmitglieder gegenüber der Digitalisierung im eigenen Unternehmen und die damit einhergehenden Konflikte insofern einen negativen Einfluss, als das sie das Aufkommen eines Prozesses zur Diffusion von digitalen Technologien und Trends unterdrücken.

Solche unterschiedlichen Ansichten der einzelnen Familienmitglieder in Bezug auf die Digitalisierung machen es erforderlich, das familiäre Werte- und Zielsystem anzugleichen. Im Allgemeinen verfügt die Familie über ein solches Werte- und Zielsystem. In diesem kommen von allen Familienmitgliedern gemeinsam geteilte Visionen, Ziele, Strategien sowie Wünsche und Präferenzen hinsichtlich der Ausrichtung des eigenen Familienunternehmens zum Ausdruck (Sieger und Zellweger 2012; Von Schlippe et al. 2017; Wimmer et al. 2011). Die Erarbeitung einer gemeinsamen familiären Haltung gegenüber dem Thema der Digitalisierung und der Integration dieser Haltung in das bestehende Wertesystem ist eine wesentliche Voraussetzung für das Zustandekommen einer Diffusion von digitalen Technologien und Trends im Familienunternehmen. Allerdings ist eine Angleichung des Wertesystems häufig nicht einfach. Insbesondere dann, wenn die Anzahl der Mitglieder im Familienkreis hoch ist und somit die Wahrscheinlichkeit für das Auftreten unterschiedlicher Ansichten steigt, müssen alle Individualmeinungen berücksichtigt und oftmals in langwierigen Prozessen miteinander in Einklang gebracht werden.

\section{$5 \quad$ Fazit}

Die Diffusion von digitalen Technologien und Trends in Familienunternehmen ist maßgeblich durch die besondere Rolle der Familie und ihren Einfluss auf das Familienunternehmen - zum Beispiel in ihrer Rolle als Eigentümer des Familienunternehmens - bestimmt. Die Geschwindigkeit, mit der bestimmte digitale Technologien und Trends 
Tab. 1 Eigenschaften der Familie, der digitalen Technologien bzw. Trends sowie des Familienunternehmens als mögliche Einflussfaktoren auf die Durchlaufgeschwindigkeit des Diffusionsprozesses. (Quelle: Eigene Darstellung)

\begin{tabular}{|c|c|c|}
\hline Eigenschaften der Familie & $\begin{array}{l}\text { Eigenschaften der } \\
\text { digitalen Technologie } \\
\text { bzw. des digitalen } \\
\text { Trends }\end{array}$ & $\begin{array}{l}\text { Eigenschaften des } \\
\text { Familienunternehmens }\end{array}$ \\
\hline $\begin{array}{l}\text { - Vorwissen der einzelnen } \\
\text { Familienmitglieder in Bezug auf } \\
\text { digitale Technologie bzw. digitalen } \\
\text { Trend } \\
\text { - Anzahl der Mitglieder in der Familie } \\
\text { - Unsicherheiten in Bezug auf digitale } \\
\text { Technologie bzw. digitalen Trend } \\
\text { - Offenheit gegenüber der } \\
\text { Digitalisierung }\end{array}$ & $\begin{array}{l}\text { - Komplexität } \\
\text { - Kompatibilität } \\
\text { - Nutzen bzw. Vorteil }\end{array}$ & $\begin{array}{l}\text { - Ressentiments der } \\
\text { Mitarbeiter gegenüber } \\
\text { der Digitalisierung }\end{array}$ \\
\hline
\end{tabular}

den Diffusionsprozess durchlaufen, hängt dabei von Eigenschaften in Bezug auf die Familie, aber auch in Bezug auf die zu Grunde liegende digitale Technologie (bzw. digitaler Trend) sowie in Bezug auf das Familienunternehmen ab, indem sie hemmend auf den Durchlauf wirken. Tab. 1 fasst die Eigenschaften der Familie, des Familienunternehmens sowie der digitalen Technologie (bzw. digitaler Trend), die als negative Einflussfaktoren auf die Durchlaufgeschwindigkeit des Diffusionsprozesses wirken, zusammen.

Je ausgeprägter sich die beschriebenen Eigenschaften darstellen, desto mehr Zeit nimmt der Durchlauf des Diffusionsprozesses in Anspruch. Dies ist als umso kritischer zu sehen, wenn das Familienunternehmen einen gewissen Marktdruck in Bezug auf die Digitalisierung zu spüren bekommt. Wenn beispielsweise die Konkurrenz bereits Effizienzvorteile durch erfolgreiche Prozessdigitalisierungen verbuchen kann und diese Effizienzvorteile als Wettbewerbsvorteile gegenüber dem Familienunternehmen durchsetzt, kann dies die wirtschaftliche Situation des Familienunternehmens mittel- bis langfristig negativ beeinflussen. Das Familienunternehmen gerät insofern unter Zugzwang, als das es diesen Wettbewerbsvorteil der Konkurrenz durch ähnliche Digitalisierungsbemühungen auszugleichen versuchen muss. Eine langsame Diffusion einer digitalen Technologie (bzw. digitaler Trend) kann darüber hinaus auch im Kontrast zu den hohen Innovationsgeschwindigkeiten, derer digitale Technologien und Trends unterliegen, stehen. Bevor die Entscheidung für die Implementierung dieser digitalen Technologie (bzw. digitaler Trend) gefallen ist, ist diese Technologie womöglich schon veraltet oder es sind inzwischen kostengünstigere Alternativen auf den Markt gekommen. Um die Diffusion von digitalen Technologien und Trends in möglichst geringer Zeit zu bewerkstelligen, nutzen Familien in der Regel die oben beschriebenen Strategien.

Andererseits muss ein langsamer Durchlauf des Diffusionsprozesses nicht nur Nachteile mit sich bringen. So kann ein höherer Zeitaufwand für die Diffusion auch bedeuten, dass die im Fokus einer Diffusion stehende digitale Technologie (bzw. digitaler Trend) sehr viel sorgfältiger in Augenschein genommen und geprüft wurde, 
was wiederum das Investitionsrisiko für diese digitale Technologie (bzw. digitaler Trend) verringert.

\section{Literatur}

Anderson, R. C., und D. M. Reeb. 2004. Board composition: Balancing family influence in S\&P 500 firms. Administrative Science Quarterly 49(2): 209-237.

Astrachan, J. H., und M. C. Shanker. 2003. Family businesses' contribution to the U.S. economy: A closer look. Family Business Review 16(3): 211-219.

Berrone, P., C. Cruz, und L. R. Gomez-Mejia. 2012. Socioemotional wealth in family firms: Theoretical dimensions, assessment approaches, and agenda for future research. Family Business Review 25(3): 258-279.

Bharadwaj, A., O. A. El Sawy, P. A. Pavlou, und N. Venkatraman. 2013. Digital business strategy: Toward a next generation of insights. MIS Quarterly 37(2): 471-482.

Blohm, I., und U. Bretschneider. 2016. Crowdsourcing. In Enzyklopädie der Wirtschaftsinformatik, Hrsg. N. Gronau, J. Becker, N. Kliewer, J. M. Leimeister und S. Overhage. http://www. enzyklopaedie-der-wirtschaftsinformatik.de/. Zugegriffen am 15.05.2019.

Bretschneider, U., A. K. Heider, T. Rüsen, und M. Hülsbeck. 2019. Strategien der Digitalisierung in Familienunternehmen: Über spezifische Digitalisierungsansätze für Unternehmerfamilien und Familienunternehmen. Witten/Deutschland: Praxisleitfaden des Wittener Instituts für Familienunternehmen (WIFU).

Campopiano, G., und A. De Massis. 2015. Corporate social responsibility reporting: A content analysis in family and non-family firms. Journal of Business Ethics 129(3): 511-534.

Chrisman, J. J., J. H. Chua, und P. Sharma. 2005. Trends and directions in the development of a strategic management theory of the family firm. Entrepreneurship: Theory \& Practice 29(5): 555-575.

Chrisman, J. J., J. H. Chua, A. W. Pearson, und T. Barnett. 2012. Family involvement, family influence, and family-centered non-economic goals in small firms. Entrepreneurship: Theory \& Practice 36(2): 267-293.

Chua, J. H., J. J. Chrisman, und P. Sharma. 1999. Defining the family business by behavior. Entrepreneurship: Theory \& Practice 23(4): 19-39.

Corsten, H., R. Gössinger, G. Müller-Seitz, und H. Schneider. 2016. Grundlagen des Technologieund Innovationsmanagements. München: Franz Vahlen.

Demary, V., B. Engels, K.-H. Röhl, und C. Rusche. 2016. Digitalisierung und Mittelstand: Eine Metastudie. IW-Analyse Nr. 109. Köln: Institut der Deutschen Wirtschaft.

Eßer, T. 2018. Die Wirkung von nicht-finanziellen Zielen auf die Vertrauenswürdigkeit von Familienunternehmen aus der Sicht ihrer Fach- und Führungskräfte. Wiesbaden: Springer Gabler.

Handler, W. C. 1989. Methodological issues and considerations in studying family businesses. Family Business Review 2(3): 257-276.

Haunschild, L., und H.-J. Wolter. 2010. Volkswirtschaftliche Bedeutung von Familien- und Frauenunternehmen. IfM-Materialien Nr. 199. Bonn: Institut für Mittelstandsforschung Bonn.

Hehn, S., N. Cornelissen, und C. Braun. 2016. Kulturwandel in Organisationen: Ein Baukasten für angewandte Psychologie im Change-Management. Berlin/Heidelberg: Springer.

Koeberle-Schmid, A., und O. Nützel. 2005. Family Business Governance: Herausforderungen und Mechanismen. INTES Zentrum für Familienunternehmen Forschungspapier Nr. 1. Vallendar: WHU Otto Beisheim School of Management.

Palfrey, J., und U. Gasser. 2008. Born digital: Understanding the first generation of digital natives. New York: Basic Books.

Pittino, D., F. Visintin, T. Lenger, und D. Sternad. 2016. Are high performance work practices really necessary in family SMEs? An analysis of the impact on employee retention. Journal of Family Business Strategy 7(2): 75-89. 
Rogers, E. M. 2003. Diffusion of innovations, 5. Aufl. New York: Free Press.

Rüsen, T., A. Heider, M. Hülsbeck, und U. Bretschneider. 2019. Die digitalisierte Unternehmerfamilie. Zeitschrift für Familienunternehmen und Strategie 9(3): 90-95.

Scheer, A. W. 2016. Thesen zur Digitalisierung. In Was treibt die Digitalisierung? Hrsg. I. Abolhassan, 49-61. Wiesbaden: Springer Gabler.

Schlaadt, C. 2017. Familienunternehmen 4.0 - Wie digitalisiert sind deutsche (Familien-)Unternehmen?, 29-37. Friedrichshafen: zupFIF, Schriftenreihe des Friedrichshafener Instituts für Familienunternehmen.

Schmidt, G. 2000. Einführung in die Organisation: Modelle, Verfahren, Techniken. Wiesbaden: Springer Gabler.

Sieger, P., und T. Zellweger. 2012. Vom Familienunternehmen zur Unternehmerfamilie. Gallen/ Schweiz.: Credit Suisse AG.

Spitzley, D., und R. Prügl. 2017. „Hört zu, seid offen, schenkt Vertrauen und lasst dem neuen Schwung Raum“: Digitalisierung in deutschen Familienunternehmen aus Sicht der nächsten Generation, 47-54. Friedrichshafen: zupFIF, Schriftenreihe des Friedrichshafener Instituts für Familienunternehmen.

Stief, S. E., A. T. Eidhoff, und M. Voeth. 2016. Transform to succeed: An empirical analysis of digital transformation in firms. International Journal of Economics and Management Engineering 10(6): 1833-1842.

Schlippe, A. von, Groth, T., und Rüsen, T. A. (2017): Die beiden Seiten der Unternehmerfamilie. Familienstrategien über Generationen. Auf dem Weg zu einer Theorie der Unternehmerfamilie. Göttingen: Vandenhoeck \& Ruprecht.

Wills, D. 2017. Same passion, different paths: How the next generation of family business leaders are making their mark. https://www.pwc.com/gx/en/family-business-services/assets/next-genstudy-2017.pdf. Zugegriffen am 08.01.2019.

Wimmer, R. 2011. Typische Schwächen und Potenziale der Selbstgefährdung von Familienunternehmen. In Familienunternehmen erfolgreich sanieren: Der Einfluss des Familienfaktors bei Restrukturierungen, Hrsg. T. Rüsen, 43-61. Berlin: Erich Schmitt.

Wimmer, R., E. Domayer, M. Oswald, und G. Vater. 2011. Familienunternehmen: Auslaufmodell oder Erfolgstyp? 2. Aufl. Wiesbaden: Springer Gabler.

Witt, P. 2008. Corporate Governance in Familienunternehmen. Zeitschrift für Betriebswirtschaft 2: $1-19$. 